\title{
Eosinophilic granulomatosis with polyangiitis (EGPA) and PRES: a case-based review of literature in ANCA-associated vasculitides
}

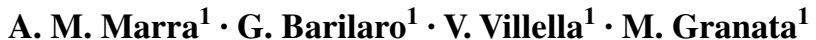

Received: 27 January 2015 / Accepted: 20 March 2015 / Published online: 3 April 2015

(C) Springer-Verlag Berlin Heidelberg 2015

\begin{abstract}
Eosinophilic granulomatosis with polyangiitis (EGPA) is a small-sized vessel systemic necrotizing vasculitis and belongs to the family of antineutrophil cytoplasmic antibody (ANCA)-associated vasculitides. The involvement of central nervous system in this condition is pretty rare. Posterior reversible encephalopathy syndrome (PRES) is a clinical and radiological entity described for the first time by Hinchey et al. (N Engl J Med 334(8):494-500, 1996) and characterized by MRI findings of reversible subcortical vasogenic edema predominantly in the white matter of posterior cerebral lobes. There are few case reports describing the concurrence of PRES with ANCA-associated vasculitides. We describe a case of PRES in a patient with a diagnosis of EGPA with a concise review of the literature. The exact cause of this syndrome is unknown. It has been related to eclampsia, drug-induced hypertension, renal insufficiency and also to rheumatologic diseases. Endothelial injury, hypertension and immunosuppressive medications can compromise the regulation of cerebral blood flow. In ANCA-associated vasculitides, patients presenting with symptoms of PRES represent a challenge to treatment with immunosuppressive medications. However, since an inflammatory process might be implicated, judicious use of these agents along with tight control of blood pressure and a supportive therapy may contribute to the resolution of the encephalopathic syndrome treating at the same time other manifestation related to the rheumatologic disease. Larger
\end{abstract}

A. M. Marra

alessandro.marra@uniroma1.it

1 Allergy and Clinical Immunology Unit, Dipartimento di Medicina Clinica - Policlinico Umberto I, Sapienza Università di Roma, Viale dell'Università 37, 00100 Rome, Italy clinical studies are warranted to optimize the management of vasculitis-associated PRES.

Keywords Eosinophilic granulomatosis with polyangiitis (EGPA) - ANCA · Vasculitis · Posterior reversible encephalopathy syndrome (PRES)

\section{Introduction}

Churg-Strauss syndrome, recently renamed eosinophilic granulomatosis with polyangiitis (EGPA), is a smallsized vessel systemic necrotizing vasculitis that was first described in the early 1950s. It belongs to the family of antineutrophil cytoplasmic antibody (ANCA)-associated vasculitides. It is a rare disease, with an annual incidence ranging between 0.5 and 6.8 per million inhabitants and a prevalence of 10.7-14 per million inhabitants [1], with a mean age at the onset around 50 years and no sex preponderance. The most typical clinical presentation of EGPA is the appearance of vasculitic manifestations in a patient with known allergic rhinitis, nasal and sinus polyposis, and late-onset asthma (almost constant, and usually preexisting for 5-10 years). General symptoms (fever or weight loss), mononeuritis multiplex, and/or necrotic cutaneous purpura are the most frequent manifestations at disease onset, in combination with elevated blood eosinophilia. The detection of ANCA, namely P-ANCA (with perinuclear labeling pattern in indirect immunofluorescence), with antimyeloperoxidase (anti-MPO) specificity in enzyme-linked immunosorbent assay, strongly supports the diagnosis, but they are present only in 35-40\% of patients [2]. The involvement of central nervous system is pretty rare. PRES (posterior reversible encephalopathy syndrome) is a clinical and radiological entity described for the first time by 
Hinchey et al. [4] and characterized by MRI findings of reversible subcortical vasogenic edema predominantly in the white matter of posterior cerebral lobes. The authors named this condition "Reversible Posterior Leukoencephalopathy Syndrome." However, some authors considered this term as misleading, as they observed also the involvement of the gray matter, hence the definition "posterior reversible encephalopathy syndrome" (PRES) was coined. Main clinical features are seizures, headache, visual disturbances and altered mental status. The cause of this syndrome is unknown. Nevertheless it has been etiologically related to hypertension (there is in fact a strong association with preeclampsia), end-stage renal disease, immunosuppressive drugs (mainly cyclosporine and tacrolimus), and rheumatologic diseases, mainly SLE, and, less frequently, Takayasu arteritis, systemic sclerosis, polyarteritis nodosa, granulomatosis with polyangiitis, thrombotic thrombocytopenic purpura. Endothelial disfunction and altered cerebral vasoregulation are implicated in the pathophysiology. There are few case reports describing the concurrence of PRES with ANCA-associated vasculitides. Here we describe a case of PRES in a patient with a diagnosis (according to the ACR criteria) of eosinophilic granulomatosis with polyangiitis (EGPA), along with a concise review of the literature. To our knowledge, this is the first documented case of PRES in a patient with a definite diagnosis of EGPA.

\section{Materials and methods}

The review was based on a literature search for articles concerning PRES in ANCA-associated vasculitides, published from 2001 through December 2014. No cases have been published before 2001. We used the Medline database (PubMed, National Library of Medicine, Bethesda, MD; keywords: posterior reversible encephalopathy, ANCA, vasculitis, connective tissue diseases). The reference lists of all articles were scanned for references not identified in the initial research. Eight cases of PRES along with ANCAassociated vasculitides were included in this study, so that with our case, we reviewed a total of 9 cases.

\section{Case}

A 40-year-old Caucasian man presented with sudden loss of strength in the four limbs and skin lesions of the lower limbs. His medical history was remarkable for asthma and nasal polyposis. He firstly developed weakness of the right hand and, 2 weeks later, numbness and weakness of the lower limbs along with the onset of vesiculous bullous skin lesions on both legs. At the admission, the patient was conscious, oriented, and had no signs of cognitive impairment. His physical examination was unremarkable except for numbness and loss of strength in the four limbs and vesiculous bullous skin lesions of the lower limbs. The patient's blood pressure was $140 / 90 \mathrm{mmHg}$. His heart rate was $72 \mathrm{bpm}$. Routine blood tests showed leukocytosis with hypereosinophilia (WBC $21,480 / \mathrm{mcl}$ with a reference range of $4000-1000$ cells $/ \mu 1$, Eos $11,190 / \mu$ with a reference range of $50-500 / \mu \mathrm{l})$. C-reactive protein was $9,94 \mathrm{mg} / \mathrm{dl}$ (normal values up to $0.5 \mathrm{mg} / \mathrm{dl}$ ), erythrocyte sedimentation rate (ESR) was $61 \mathrm{~mm} / \mathrm{h}$ (normal values up to $15 \mathrm{~mm} / \mathrm{h}$ ), and p-ANCA were positive at high titer (100 $\mathrm{UA} / \mathrm{ml}$ ). Blood urea nitrose was normal and his serum creatinine was $0.7 \mathrm{mg} / \mathrm{dL}$. A brain MRI and MRI of the spine performed before being admitted to our department showed no significant abnormalities. A chest X-ray showed pulmonary infiltrates in the right lung. An electroneurography showed sensori-motor axonal neuropathy with asymmetric distribution (mononeuritis multiplex), compatible with vasculitis. A skin biopsy and muscle biopsy showed vasculitis with extravascular eosinophils. Eosinophilic granulomatosis with polyangiitis was diagnosed as the patient fulfilled 5 of the 6 criteria by the American College of Rheumatology. Serum creatinine was normal, and he had no renal damage. Blood pressure was in normal range at the exordium. The patient was treated with intravenous methylprednisolone (1 gram per day for 3 days), followed by prednisone $1 \mathrm{mg} / \mathrm{kg}$ with subsequent tapering. A week after starting treatment, he presented generalized tonic-clonic seizures, headache, and transient lack of vision. His blood pressure at the onset of crisis was 170/100 mmHg. A brain MRI was performed, and T2-weighted image showed bilateral high-intensity areas in the white matter of the occipital and parietal lobes. These findings (Fig. 1) were suggestive of PRES (posterior reversible encephalopathy syndrome). The patient was treated with antihypertensive drugs and diazepam with benefit. A brain MRI performed 10 days later showed partial resolution of radiological findings, which were no more detectable on a new MRI one month later. Prednisone was tapered further, and azathioprine was added as steroid sparing drug. He did not show any other symptom of PRES afterward.

\section{Results}

Only eight cases of PRES in patients with ANCA-associated vasculitis have been reported in the literature (Table 1). Seven of these are isolated case reports. One has been included in a larger review of 18 cases of PRES associated with connective tissue diseases [3]. Among the 9 cases of PRES linked to ANCA-associated vasculitis reported to date (including ours), 3 were males and 6 were females, with a mean age of 47.7 years (range 14-77 y.o.). In all 


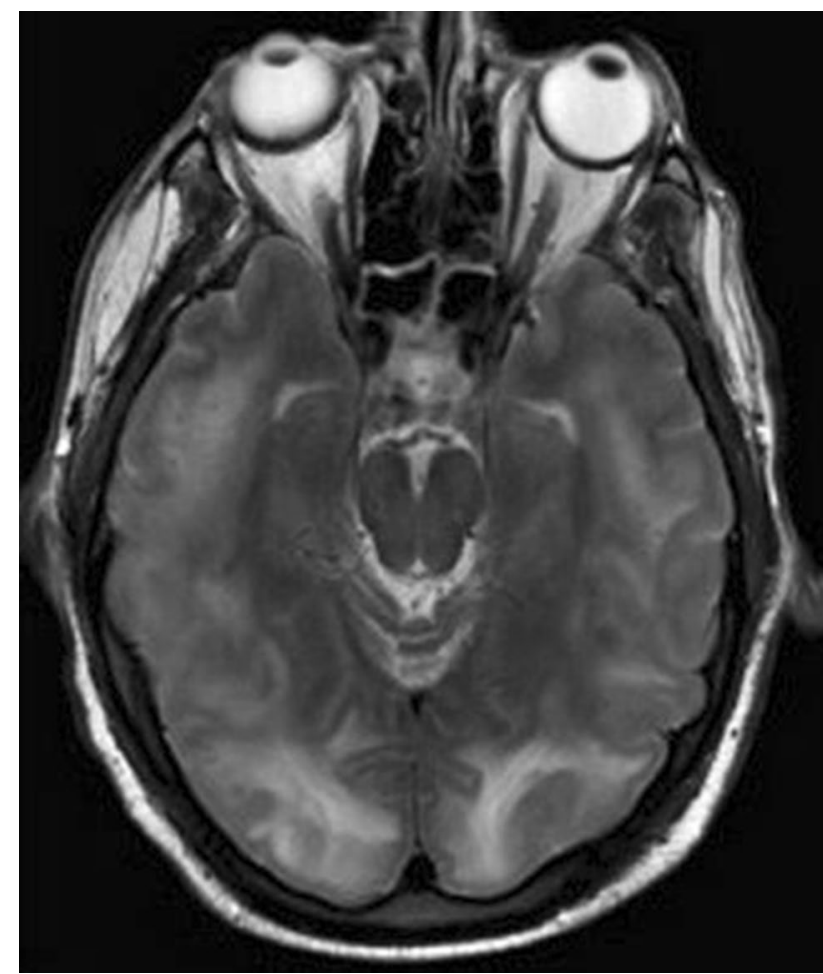

Fig. 1 Bilateral high-intensity areas in the white matter of the occipital and parietal lobes in MRI T2-weighted image. These findings were suggestive of PRES

cases, the diagnosis of PRES was made on the basis of MRI findings. Six patients presented with severe systemic hypertension, one with moderate systemic hypertension, and two with normal blood pressure. Three patients had an underlying diagnosis of granulomatosis with polyangiitis
(GPA), 2 patients had microscopic polyangiitis (MPA), 3 had an ANCA-associated vasculitis (namely p-ANCA) with no sufficient criteria to make a diagnosis of EGPA, GPA, or MPA, and our patient was the only one with a definite diagnosis of EGPA. Serum creatinine value was increased in 4 patients. In all cases, PRES was diagnosed by the typical findings of high-signal intensity areas on T2-weighted fluid-attenuated inversion-recovery images (FLAIR) and low signal intensity on T1-weighted images in the same lesions. Eight of nine patients were taking corticosteroids at the onset of symptoms of PRES. Three of them were also receiving cyclophosphamide (CY) because of the severity of the disease. None of them stopped the steroid treatment, and only one patient stopped CY. In 4 patients, a methylprednisolone pulse therapy was started, as PRES was considered a manifestation of the underlying immunologic disease. In one case, the diagnosis of PRES preceded the diagnosis of the ANCA-associated vasculitis; hence, PRES was the first manifestation of the disease. In our case, a control MRI 10 days after the onset of symptoms showed a significant improvement in the radiological findings, and repeated MRI 1 month after the initial severely abnormal study showed a complete resolution of the affected areas and the patient had a full neurological recovery.

\section{Discussion}

PRES is a clinical and radiological entity described for the first time by Hinchey et al. [4]. The main clinical symptoms and signs are headache, decreased alertness, and behavior ranging from drowsiness to stupor, seizures, vomiting, mental abnormalities including confusion, diminished

Table 1 Main features of the nine cases reviewed

\begin{tabular}{|c|c|c|c|c|c|c|}
\hline References & Age/sex & Underlying disease & Medications & PRES treatment & Blood pressure & Creatinine \\
\hline Primavera [7] & $23 \mathrm{M}$ & GPA & mPSLpulse/CY & CY withdrawn & $200 / 150 \mathrm{mmHg}$ & $3.6 \mathrm{mg} / \mathrm{dl}$ \\
\hline Kawano [18] & $73 \mathrm{~F}$ & MPA & mPSLpulse & mPSLpulse & $200 / 100 \mathrm{mmHg}$ & $0.9 \mathrm{mg} / \mathrm{dl}$ \\
\hline Kawano [18] & $77 \mathrm{~F}$ & MPA & mPSLpulse & mPSLpulse & $180 / 120 \mathrm{mmHg}$ & $3 \mathrm{mg} / \mathrm{dl}$ \\
\hline Ohta [8] & $14 \mathrm{~F}$ & GPA & $\begin{array}{l}\mathrm{PSL}+\mathrm{PE}+\mathrm{CY} \text { (with- } \\
\text { drawn at } 6 \text { wks because } \\
\text { of leucopenia) }\end{array}$ & No changes & $180 / 90 \mathrm{mmHg}$ & $7.8 \mathrm{mg} / \mathrm{dl}$ \\
\hline Tajima [12] & $76 \mathrm{~F}$ & p-ANCA ass. vasc. & PSL & mPSLpulse & $130 / 80 \mathrm{mmHg}$ & $0.8 \mathrm{mg} / \mathrm{dl}$ \\
\hline Nishio [16] & $15 \mathrm{~F}$ & GPA & PSL- mPSLpulse- CY & mPSLpulse-CY & $120 / 60 \mathrm{mmHg}$ & $0.27 \mathrm{mg} / \mathrm{dl}$ \\
\hline Fuentes [11] & $72 \mathrm{~F}$ & $\begin{array}{l}\text { GPA (?) p-ANCA ass. } \\
\text { vasc. }\end{array}$ & PSL & PSL only & $150 / 100 \mathrm{mmHg}$ & n.a. \\
\hline Patel [10] & $40 \mathrm{M}$ & p-ANCA & $\begin{array}{l}\text { Hemodialysis, mPSL } \\
\text { pulses + PSL }\end{array}$ & Dialysis, mPSL- PSL & $160 / 90 \mathrm{mmHg}$ & $24.48 \mathrm{mg} / \mathrm{dl}$ \\
\hline $\begin{array}{l}\text { Marra Barilaro } \\
\text { (2014) }\end{array}$ & $40 \mathrm{M}$ & EGPA & mPSL e PSL & PSL only & $140 / 90 \mathrm{mmHg}$ & $0.7 \mathrm{mg} / \mathrm{dl}$ \\
\hline
\end{tabular}

GPA granulomatosis with polyangiitis, EGPA eosinophilic granulomatosis with polyangiitis, MPA microscopic polyangiitis, $p$-ANCA ass. vasc. P-ANCA-associated vasculitis with no sufficient criteria to make a diagnosis of GPA, MPA, EGPA, $m P S L$ methylprednisolone, $P S L$ prednisolone, $P E$ plasma exchange, $C Y$ cyclophosphamide 
spontaneity and speech, and abnormalities of visual perception like visual loss, including cortical blindness. The onset is usually subacute but may be heralded by a seizure. Seizures are common at the onset of neurologic symptoms but can also develop later. Seizures may begin focally but usually become generalized. Multiple seizures are more common than single events. Stupor and frank coma may develop, but usually patients remain responsive to stimuli [5].

The most common abnormality on neuroimaging is vasogenic edema involving the white matter in the posterior portions of the cerebral hemispheres, especially in the temporo-parieto-occipital regions, bilaterally. However, involvement of additional areas of the brain has also been reported. Computed tomography findings consist of bilaterally symmetric low attenuation in the posterior parietal/ occipital lobes. MRI, particularly diffusion-weighted MRI, is the most appropriate radiologic exam and may help in discriminating the vasogenic edema of PRES from the cytotoxic edema of early cerebral infarction [6]. Typically vasogenic edema is represented by hyperintensity in fluid-attenuated inversion-recovery and T2-weighted MRI, while DWI sequences are normal, and the apparent diffusion coefficient map shows increased activity, the exact opposite to what would be expected in brain stroke. This is a really important feature in differentiating the two conditions. Although posterior changes are prominent in this syndrome, frontal involvement is more frequent than posterior on FLAIR imaging and DWI.

The exact pathogenesis of the posterior reversible encephalopathy syndrome is not perfectly known. It is associated with many conditions causing hypertensive encephalopathy, as eclampsia, drug-induced hypertension, and renal insufficiency. PRES has also been reported in association with many rheumatologic diseases.

Rheumatologic diseases can have varied clinical presentations involving the central nervous system. SLE has been the first autoimmune disease linked to PRES [7], but, since then, several case reports described the association between PRES and Takayasu arteritis, systemic sclerosis, polyarteritis nodosa, Henoch-Schönlein purpura, and granulomatosis with polyangiitis.

The differential diagnosis includes multiple embolic lesions and vertebrobasilar thrombo-occlusive disease, but also widespread inflammatory diseases with involvement of the central nervous system (CNS) such as neuro-SLE and primary angiitis of the CNS. Patients with PRES and patients with CNS vasculitis may have a similar clinical presentation characterized by encephalopathy, visual loss, seizures, and headache. However, CNS vasculitis lacks the typical MRI findings of PRES, which is helpful in distinguishing the first condition that might require aggressive immunosuppressive therapy from the second that does not.
A possible explanation of this phenomenon is the endothelial injury caused by an inflammatory state, vasculitis, or anti-endothelial antibodies that could contribute to the disruption of the blood-brain barrier and lead to cerebral vasogenic edema. In particular, in ANCA-associated vasculitides, ANCA could by itself activate neutrophils causing the endothelial cell injury, leading to the development of PRES [8]. This can also explain the efficacy of strong immunosuppressive therapy as treatment of PRES in patients with ANCA-associated vasculitis [3]. However, endothelial injury alone is likely not sufficient to cause PRES and a second factor, namely hypertension, can lead to alterations in cerebral blood flow regulation. In particular, hypertension causes increased capillary filtration, and sudden elevations in systemic blood pressure can exceed the autoregulatory capacity of the brain vasculature. Regions of vasodilation and vasoconstriction develop, especially in arterial boundary zones, and there is a breakdown of the blood-brain barrier with focal transudation of fluid and petechial hemorrhages. Hence it is likely that at least two mechanisms are involved in the development of PRES, namely hypertension and endothelial injury, both leading to altered cerebral autoregulation. [9].

Only in one case of the 9 reported so far (Table 1), PRES was the onset manifestation of the undergoing vasculitis [10] (the involvement of CNS in ANCA-associated vasculitides is rare). All remaining patients had a previous diagnosis of ANCA-associated vasculitis and were under steroid treatment at the onset of PRES. In particular, five patients were treated with methylprednisolone pulse therapy, in two cases associated with cyclophosphamide, one patient with oral methylprednisolone and cyclophosphamide [3], and two with oral methylprednisolone alone $[11,12]$. Remarkably, no patient stopped the corticosteroid therapy after the onset of PRES, even those who presented with severe hypertension. In four patients, PRES was successfully treated with methylprednisolone pulse therapy, supporting the hypothesis of PRES as a neurologic manifestation of the underlying rheumatologic disease.

Immunomodulatory drugs have also been associated with the development of PRES. The pathophysiology of this phenomenon is not completely understood, but it can be explained by a direct effect on the cerebral vasculature causing a capillary leak leading to the cerebral vasogenic edema. Another possible mechanism is vasoconstriction caused by overproduction of endothelin. Drugs most frequently associated with PRES are the calcineurin inhibitors, cyclosporine, and tacrolimus [13]. However, cases of PRES have also been described in association with mycophenolate mofetil, methotrexate [14], and etanercept [15]. Corticosteroids represent the first-line therapy in ANCA-associated vasculitides. All patients reviewed in this article, except one, received steroid treatment 
before presenting PRES. This effect can be explained by an increase in systemic blood pressure caused by CS. Moreover, corticosteroid therapy has been shown to inhibit endothelial prostacyclin production and to increase thromboxane A2 production, leading to platelet aggregation and to a decrease in blood flow to the organs. A decreased blood flow in the kidney activates the reninangiotensin system leading to a rapid elevation in blood pressure and precipitating endothelial dysfunction [16]. It is likely that in our patient steroid therapy, increasing the blood pressure could have worked as a trigger in a predisposed substrate represented by the endothelial injury due to the vasculitis.

The treatment of PRES is mainly based on reducing blood pressure. Antiepileptic drugs should be used to control acute seizures. Most case series suggest the PRES is usually a benign condition, but neurologic sequelae have been reported with evolution of the initial vasogenic edema into cytotoxic edema, infarction, or hemorrhage [17]. However, complete resolution of neurological symptoms along with improvement or resolution of imaging alterations occurs within few days to weeks of presentation, if a prompt control of blood pressure is started along with the removal of inciting factors.

In our case, the patient was under oral prednisone at PRES onset. We decided not to stop or modify the steroid therapy and started aggressive blood pressure control with carvedilol and clonidine. We also started intravenous diazepam as antiepileptic therapy. However, other authors, as Kawano and Nishio [18], [16], considered PRES as a neurologic complication of the underlying vasculitis and promptly started methylprednisolone intravenous pulse therapy.

In ANCA-associated vasculitides, as other autoimmune diseases, patients presenting with symptoms of PRES and major organ involvement represent a challenge to treatment with immunosuppressive medications, especially considering that some of these therapies have been associated with the development of PRES. However, since an inflammatory process might be implicated, judicious use of these agents along with aggressive blood pressure control and supportive therapy may contribute to the resolution of the encephalopathic syndrome providing at the same time adequate control of other organs involvement. In conclusion, physicians should be aware that immunosuppressive therapy can be a trigger of PRES, but, especially in severe cases where the immunologic disease is life threatening, stopping immunosuppression is not advisable. Larger clinical studies, made difficult by the rarity of both conditions, are warranted to optimize the management of vasculitis-associated PRES.

Conflict of interest The authors declare no conflict of interests.

\section{References}

1. Pagnoux C, Guilpain P, Guillevin L (2007) Churg-Strauss syndrome. Curr Opin Rheumatol 19(1):25-32

2. Pagnoux C (2010) Churg-Strauss syndrome: evolving concepts. Discov Med 9(46):243-252

3. Min L, Zwerling J, Ocava LC, Chen IH, Putterman C (2006) Reversible posterior leukoencephalopathy in connective tissue diseases. Semin Arthritis Rheum 35(6):388-395

4. Hinchey J, Chaves C, Appignani B, Breen J, Pao L, Wang A, Pessin MS, Lamy C, Mas JL, Caplan LR (1996) A reversible posterior leukoencephalopathy syndrome. N Engl J Med 334(8):494-500

5. Ay H, Buonanno FS, Schaefer PW, Le DA, Wang B, Gonzalez RG, Koroshetz WJ (1998) Posterior leukoencephalopathy without severe hypertension: utility of diffusion-weighted MRI. Neurology 51(5):1369-1376

6. Kastrup O, Schlamann M, Moenninghoff C, Forsting M, Goericke S (2014) Posterior reversible encephalopathy syndrome: the spectrum of MR imaging patterns. Clin Neuroradiol [Epub ahead of print]

7. Primavera A, Audenino D, Mavilio N, Cocito L (2001) Reversible posterior leucoencephalopathy syndrome in systemic lupus and vasculitis. Ann Rheum Dis 60(5):534-537

8. Ohta T, Sakano T, Shiotsu M, Furue T, Ohtani H, Kinoshita Y, Mizoue T, Kiya K, Tanaka I (2004) Reversible posterior leukoencephalopathy in a patient with Wegener granulomatosis. Pediatr Nephrol 19(4):442-444

9. Camara-Lemarroy CR, Lara-Campos JG, Perez-Contreras E, Rodríguez-Gutiérrez R, Galarza-Delgado DA (2013) Takayasu's arteritis and posterior reversible encephalopathy syndrome: a case-based review. Clin Rheumatol 32(3):409-415

10. Patel UV, Patel NJ (2014). Posterior reversible leukoencephalopathy syndrome as a presenting manifestation of p-ANCAassociated vasculitis. BMJ Case Rep 2014. doi:10.1136/ bcr-2013-202022

11. Fuentes AG, Komarla A, Gomez JI (2012) Posterior reversible encephalopathy syndrome in a patient with ANCA-associated vasculitis. Rheumatol Int 32(8):2529-2530

12. Tajima Y, Matsumoto A (2006) Reversible posterior leukoencephalopathy syndrome in p-ANCA-associated vasculitis. Intern Med 45(20):1169-1171

13. Shin KC, Choi HJ, Bae YD, Lee JC, Lee EB, Song YW (2005) Reversible posterior leukoencephalopathy syndrome in systemic lupus erythematosus with thrombocytopenia treated with cyclosporine. J Clin Rheumatol 11(3):164-166

14. Raghavendra S, Nair MD, Chemmanam T, Krishnamoorthy T, Radhakrishnan VV, Kuruvilla A (2007) Disseminated necrotizing leukoencephalopathy following low-dose oral methotrexate. Eur J Neurol 14(3):309-314

15. Kastrup O, Diener (2008) TNF-antagonist etanercept induced reversible posterior leukoencephalopathy syndrome. HCJ Neurol 255(3):452-453

16. Nishio M, Yoshioka K, Yamagami K, Morikawa T, Konishi Y, Hayashi N, Himuro K, Imanishi M (2008) Reversible posterior leukoencephalopathy syndrome: a possible manifestation of Wegener's granulomatosis-mediated endothelial injury. Mod Rheumatol 18(3):309-314

17. Covarrubias DJ, Luetmer PH, Campeau NG (2002) Posterior reversible encephalopathy syndrome: prognostic utility of quantitative diffusion-weighted MR images. AJNR Am J Neuroradiol 23(6):1038-1048

18. Kawano H, Kimura E, Ishizaki M, Nishida Y, Matsumoto N, Yamashita T, Hirano T, Uchino M (2002) Reversible posterior leukoencephalopathy syndrome in two patients with microscopic polyarteritis nodosa. Rinsho Shinkeigaku Clin Neurol 42(10):949-953 